\title{
Phosphorylation modulates rapid nucleocytoplasmic shuttling and cytoplasmic accumulation of Neurospora clock protein FRQ on a circadian time scale
}

\author{
Axel C.R. Diernfellner, ${ }^{1,3}$ Christina Querfurth, ${ }^{1,3}$ Carlos Salazar, ${ }^{2}$ Thomas Höfer, ${ }^{2}$ \\ and Michael Brunner ${ }^{1,4}$ \\ ${ }^{1}$ University of Heidelberg Biochemistry Center, 69120 Heidelberg, Germany; ${ }^{2}$ German Cancer Research Center and BioQuant \\ Center, Research Group Modeling of Biological Systems, 69120 Heidelberg, Germany
}

The Neurospora clock protein FREQUENCY (FRQ) is an essential regulator of the circadian transcription factor WHITE COLLAR COMPLEX (WCC). In the course of a circadian period, the subcellular distribution of FRQ shifts from mainly nuclear to mainly cytosolic. This shift is crucial for coordinating the negative and positive limbs of the clock. We show that the subcellular redistribution of FRQ on a circadian time scale is governed by rapid, noncircadian cycles of nuclear import and export. The rate of nuclear import of newly synthesized FRQ is progressively reduced in a phosphorylation-dependent manner, leading to an increase in the steady-state level of cytoplasmic FRQ. The long-period $f r^{7}$ mutant displays reduced kinetics of FRQ ${ }^{7}$ protein phosphorylation and a prolonged accumulation in the nucleus. We present a mathematical model that describes the cytoplasmic accumulation of wild-type and mutant FRQ on a circadian time scale on the basis of frequency-modulated rapid nucleocytoplasmic shuttling cycles.

[Keywords: FRQ; subcellular shuttling; phosphorylation; circadian clock]

Supplemental material is available at http://www.genesdev.org.

Received May 6, 2009; revised version accepted August 12, 2009.

Circadian clocks are evolutionary widespread molecular devices that coordinate expression profiles of large numbers of genes in anticipation of daily changes in environmental conditions. Eukaryotic clocks are made up of interconnected positive and negative feedback loops that generate self-sustained daily oscillations of clock protein function and abundance at transcriptional and post-transcriptional levels (Ko and Takahashi 2006; Liu and BellPedersen 2006; Dunlap et al. 2007; Rosbash et al. 2007). In nature, clocks are entrained to the 24-h period of earth rotation (Merrow and Roenneberg 2007; Roenneberg and Merrow 2007). The major components of these loops have been identified in several model organisms, and their interactions and dynamics have been established in a number of cases. Yet, we still lack a concept to explain how such feedback loops robustly and reliably produce oscillations in the circadian range.

\footnotetext{
${ }^{3}$ These authors contributed equally to this work.

${ }^{4}$ Corresponding author.

E-MAIL michael.brunner@bzh.uni-heidelberg.de; FAX 49-6221-544769.

Article is online at http://www.genesdev.org/cgi/doi/10.1101/gad.538209
}

The transcriptional activator WHITE COLLAR COMPLEX (WCC) is a central component of the circadian clock of Neurospora (Dunlap et al. 2007). It rhythmically activates, either directly or indirectly, the expression of numerous clock-controlled genes (Vitalini et al. 2004). One of these genes is the circadian regulator FREQUENCY (FRQ), which inhibits the WCC in a negative feedback loop (Aronson et al. 1994) and supports its accumulation in a positive loop (Lee et al. 2000; Cheng et al. 2001). Negative and positive feedback loops are coordinated in a temporal and spatial fashion (Schafmeier et al. 2006) and are governed by the same molecular mechanism: FRQ recruits CK1a and supports phosphorylation of the WCC (Schafmeier et al. 2005). Hyperphosphorylation of the WCC has a stabilizing effect and inactivates the transcription factor by several means (Schafmeier et al. 2008). Hypophosphorylated WCC is concentrated in nuclei, can bind DNA, and is active. However, it is highly unstable, and its degradation appears to be triggered by binding to DNA. FRQ-dependent phosphorylation of the WCC interferes with DNA binding and nuclear import (negative feedback) and, thus, with rapid turnover. 
Accordingly, when FRQ levels are high, WCC is stable, and newly synthesized WCC accumulates over the course of hours (positive feedback). Hence, rhythmically expressed FRQ supports circadian oscillations in WCC activity and stability, which in turn support rhythmic transcription of frq RNA and expression of FRQ protein (Hong et al. 2008; Schafmeier et al. 2008).

Oscillations in WCC activity and abundance, which are apparent on a circadian time scale, are governed by rapid, noncircadian cycles of phosphorylation and dephosphorylation and of nucleocytoplasmic shuttling. FRQ-dependent phosphorylation of the WCC is antagonized by the protein phosphatases PP2A (Schafmeier et al. 2005) and PP4 (Cha et al. 2008). PP2A activity is cytosolic, and so, low levels of nuclear FRQ are adequate for efficient phosphorylation and inactivation of the WCC in the nucleus (Schafmeier et al. 2008). Reactivation of WCC requires nuclear export, dephosphorylation in the cytosol, and reimport into the nucleus, which occurs over minutes. In the course of a day, FRQ is progressively phosphorylated and accumulates in the cytosol by an unknown mechanism. Like nuclear FRQ, cytosolic FRQ supports phosphorylation of the WCC and competes with dephosphorylation and reactivation. Since hyperphosphorylation of the WCC interferes with nuclear import, cytoplasmic FRQ modulates the shuttling frequency of the WCC and thereby shifts the shuttling equilibrium toward cytoplasmic localization. Hyperphosphorylation of WCC also interferes with DNA binding and degradation and, hence, inactive WCC accumulates to a high level when FRQ is expressed (Schafmeier et al. 2008). To complete a circadian cycle, hyperphosphorylated FRQ is degraded. As FRQ levels decrease, dephosphorylation of WCC dominates over FRQ-dependent phosphorylation. The WCC is progressively reactivated and imported into the nucleus where it drives a new round of frq transcription (Brunner and Kaldi 2008).

Circadian modulation of the kinetics of nucleocytoplasmic shuttling of WCC should depend on coordinated rhythmic accumulation of FRQ in the nuclei and cytosol. FRQ contains a nuclear import signal, which is essential for its function (Luo et al. 1998). Yet, it is localized predominantly in the cytosol (Schafmeier et al. 2005). The molecular mechanisms underlying the dynamics of subcellular localization of FRQ are not known.

In the current study, we developed an in vivo nucleocytoplasmic shuttling assay based on the rapamycin-induced complex formation of FK506-binding protein (FKBP) and the FKBP-rapamycin-binding domain of mTOR (FRB) (Banaszynski et al. 2005), which were fused to histone $\mathrm{H} 1$ and FRQ, respectively. We used that assay to examine the effects of phosphorylation on the relocalization of FRQ between the nucleus and cytoplasm. We also developed a mathematical model to describe the relocalization.

\section{Results}

Nucleocytoplasmic shuttling of FRQ

Despite the presence of a functional nuclear localization signal in its N-terminal portion (Luo et al. 1998), the majority of FRQ is localized in the cytosol (Schafmeier et al. 2005). We asked whether the apparent subcellular distribution of FRQ reflects the dynamics of nucleocytoplasmic shuttling. Attempts to visualize shuttling of FRQ-GFP fusion proteins in living mycelia were not successful, due to the low expression levels of FRQ-GFP (data not shown). We therefore developed a biochemical shuttling assay based on rapamycin-induced heterodimerization of FRB and FKBP (Banaszynski et al. 2005). These domains were genetically fused to the ORFs of $f r q$ and histone H1, respectively, and expressed in a $\mathrm{rr}^{10}{ }^{10}$ (frqnull) background (Supplemental Fig. 1A). The corresponding Neurospora strain is referred to as frq-frb/h1-fkbp. The system is similar to the anchor-away technique in Saccharomyces cerevisiae (Haruki et al. 2008).

FRQ-FRB was expressed under control of the frq promoter at approximately wild-type levels and localized predominantly in the cytosol (Fig. 1A). When the mycelia were incubated for $2 \mathrm{~h}$ with rapamycin, the cytosolic FRQFRB was efficiently trapped in the nucleus by rapamycininduced dimerization with H1-FKBP. Rapamycin-induced nuclear accumulation of FRQ-FRB was dependent on expression of H1-FKBP (Supplemental Fig. 1B). Rapamycin did not affect the subcellular distribution of FRQ in a wildtype strain (Fig. 1A). Similarly, the subcellular distribution of WC-1 and WC-2 were not affected by rapamycin in the frq-frb/h1-fkbp strain (Supplemental Fig. 1C). The data demonstrate that FRQ-FRB shuttles between cytosol and nuclei and that the observed nucleoytoplasmic distribution reflects the equilibrium between nuclear import and export. frq-frb/h1-fkbp was arrhythmic in constant darkness but rhythmic in light-dark (LD) and temperature cycles, suggesting that the FRQ-FRB fusion protein was partially functional. In the presence of rapamycin, frq-frb/h1-fkbp became arrhythmic in LD cycles, suggesting that shuttling of FRQ is critical for clock function (Supplemental Fig. 1D).

To assess the kinetics of shuttling, frq-frb/h1-fkbp was grown without rapamycin in constant light (LL). Under these conditions, FRQ-FRB is constitutively expressed and heterogeneously phosphorylated. Rapamycin was added, and mycelia were harvested over a period of 120 min and fractionated (Fig. 1B). Cytosolic FRQ-FRB was trapped in the nucleus within minutes, indicating that it shuttles rapidly between compartments. We noted that hypophosphorylated FRQ-FRB was trapped in the nucleus more efficiently than hyperphosphorylated species. This correlation suggests that shuttling of FRQ-FRB is regulated by phosphorylation.

\section{Shuttling kinetics and phosphorylation state of FRQ}

To determine the effect of phosphorylation on shuttling, we analyzed nuclear trapping of hypophosphorylate and hyperphosphorylated species of FRQ-FRB. To generate a pool of hypophosphorylated FRQ-FRB, dark-grown mycelia were exposed to light to rapidly induce de novo synthesis of FRQ-FRB (Fig. 2A, LI). After 2 h, rapamycin was added, and the kinetics of nuclear accumulation of FRQ-FRB was analyzed. The newly synthesized, 
A

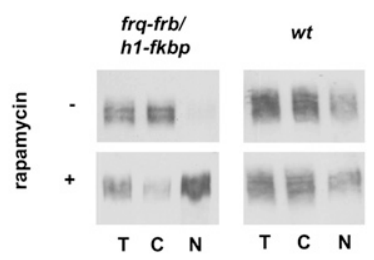

B

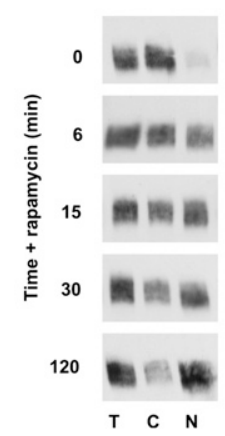

Figure 1. FRQ shuttles rapidly between cytosol and the nucleus. (A) Formation of the rapamycin-induced ternary complex of FRB-tagged FRQ with FKBP-tagged histone $\mathrm{H} 1$ in the nucleus. Mycelia of $f r q-f r b / h 1-f k b p$ and wild type were either treated with $200 \mathrm{nM}$ rapamycin for $2 \mathrm{~h}$ or left untreated. Total cell extract $(T)$, cytoplasmic $(\mathrm{C})$, and nuclear $(\mathrm{N})$ fractions were subjected to Western analysis and probed for FRQ-FRB. $(B)$ Time course of rapamycin-induced nuclear accumulation of FRQFRB. Rapamycin was added to light-grown frq-frb/h1-fkbp. Mycelia were harvested, fractionated at the indicated times, and analyzed.

hypophosphorylated FRQ-FRB was rapidly and efficiently trapped in the nucleus, demonstrating that it was importcompetent (Fig. 2B, LI).

Upon light-to-dark (LD) transfer of mycelia, synthesis of FRQ is shut down and the previously synthesized protein is progressively phosphorylated and eventually degraded. To generate a pool of FRQ-FRB with a higher overall phosphorylation state, light-grown mycelia were transferred to darkness for $6 \mathrm{~h}$ (Fig. 2A, DD6). When rapamycin was added, FRQ-FRB accumulated in nuclei (Fig. 2B, DD6). The kinetics of accumulation was, however, slower than with the LI samples, suggesting that phosphorylation of FRQ interferes with nuclear import.

To investigate further whether phosphorylation interferes with nuclear import, we produced a pool of highly phosphorylated FRQ-FRB by incubating mycelia for $2 \mathrm{~h}$ with cycloheximide (CHX) (Fig. 2A, CHX). CHX inhibits protein synthesis and induces rapid hyperphosphorylation of FRQ by an unknown mechanism (Gorl et al. 2001). The extent of CHX-induced phosphorylation of FRQ-FRB was higher than under steady-state conditions in constant light (LL). When rapamycin was added, the hyperphosphorylated FRQ-FRB remained in the cytosol (Fig. 2B, CHX). Since histone H1-FKBP was stable in the presence of CHX (Supplemental Fig.2A), the data suggest that hyperphosphorylation of FRQ-FRB inhibits nuclear accumulation. It should be noted that CHX does not affect the shuttling kinetics of WC-1 and WC-2 (Schafmeier et al. 2008), demonstrating that it does not generally inhibit nuclear trafficking. Yet, we cannot exclude that a putative factor required specifically for the nuclear localization of FRQ-FRB is rapidly degraded in the presence of CHX.

To address this issue directly, mycelia were incubated with CHX for $2 \mathrm{~h}$ in the absence or presence of the highly specific CK1 $\delta$ inhibitor PF670462 (Badura et al. 2007; Bryant et al. 2009), and then rapamycin was added (Fig. 2C; Supplemental Fig. 2B). PF670462, which efficiently inhibited recombinant Neurospora CKla in vitro (data not shown), inhibited only part of the hyperphosphorylation of FRQ-FRB in vivo in the presence of CHX (Supplemental Fig. 2B). FRQ is heterogeneously phosphorylated at $>70$ sites by a number of different kinases (Baker et al. 2009; Tang et al. 2009). Only a fraction of theses sites are targets for CK1 (Tang et al. 2009). Thus, the data suggest that PF670462 inhibited specifically CK1-dependent phosphorylation of FRQ-FRB, but did not affect phosphorylation by other kinases.

Interestingly, PF670462 efficiently prevented degradation of FRQ-FRB in the presence of CHX (Fig. 2C; Supplemental Fig. 2B), demonstrating that CK1 activity is crucial for FRQ turnover. When rapamycin was added for 15 min, a substantial fraction of FRQ-FRB was trapped in the nucleus despite the presence of CHX, suggesting that CK1-specific phosphorylation rather than CHX per se interferes with nuclear accumulation of FRQ-FRB.

Finally, to directly compare the subcellular distribution of hypophosphorylated and of hyperphosphorylated FRQFRB, we generated distinguishable pools of both phosphorylation species in the same mycelia. For this purpose light-grown mycelia were transferred to the dark until the previously synthesized FRQ-FRB had acquired a rather high phosphorylation state. Subsequently, the mycelia were exposed to light to rapidly induce synthesis of new, hypophosphorylated FRQ-FRB. Then, rapamycin was added for $15 \mathrm{~min}$ and the mycelia were fractionated. Under these conditions, hyperphosphorylated "old" and hypophosphorylated "young" FRQ-FRB can be separated by SDS-PAGE (Fig. 2D, filled and open arrowheads, respectively) and analyzed by densitometry (Fig. 2E). Quantification of 12 independent experiments revealed that newly synthesized hypophosphorylated FRQ-FRB is enriched in the nuclei in comparison with hyperphosphorylated FRQ-FRB (Fig. 2F).

We then asked whether phosphorylation affects the export of FRQ-FRB. To monitor nuclear export, FRQ-FRB was first allowed to accumulate in the nucleus by rapamycin-induced complex formation with H1-FKBP. The mycelia were then transferred to medium containing FK506, a competitor of rapamycin that binds to FKBP and dissociates the complex between the FRB and FKBP moieties (Banaszynski et al. 2005). With FK506, the H1FKBP-bound nuclear FRQ-FRB redistributed progressively to the cytosol, indicating that the protein was exported from nuclei (Supplemental Fig. 3, left). Next, we allowed FRQ-FRB to accumulate in the nucleus, and 
A

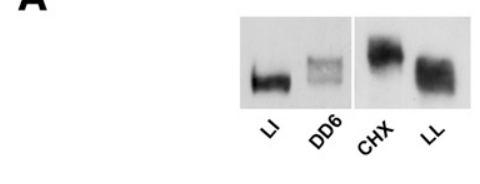

B
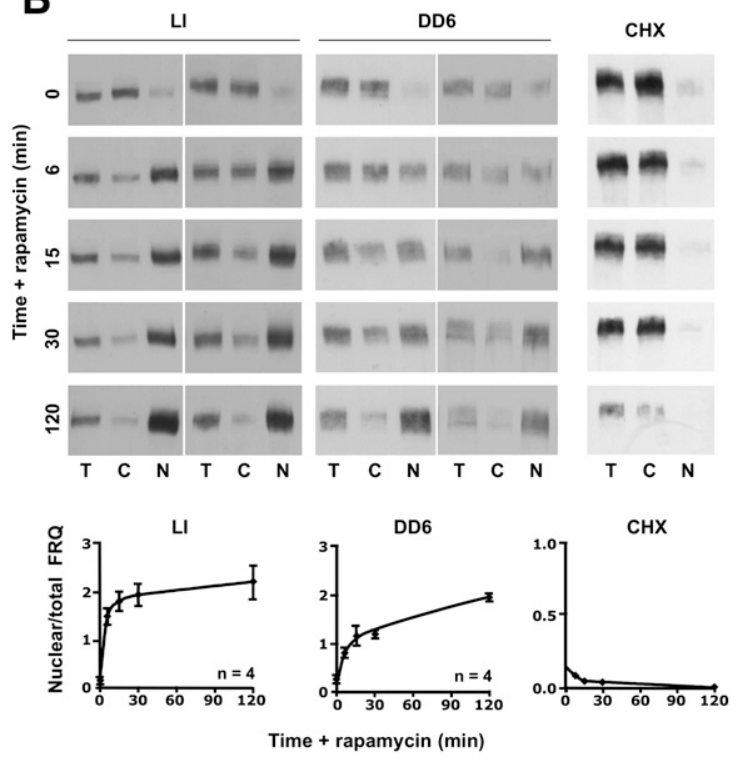

C

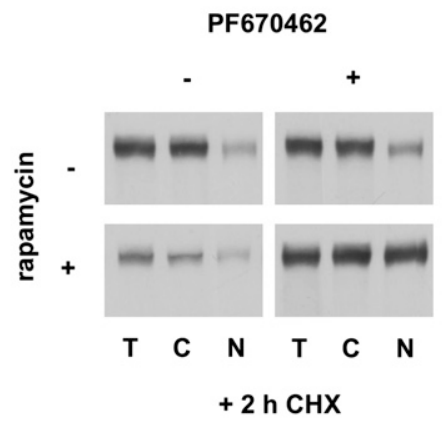

D

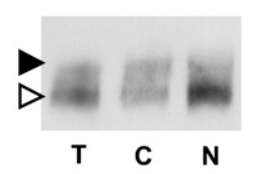

$\mathbf{F}$

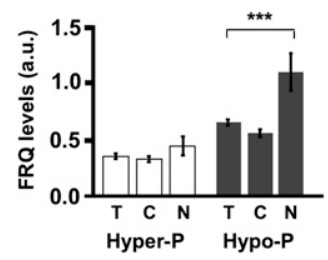

$E$

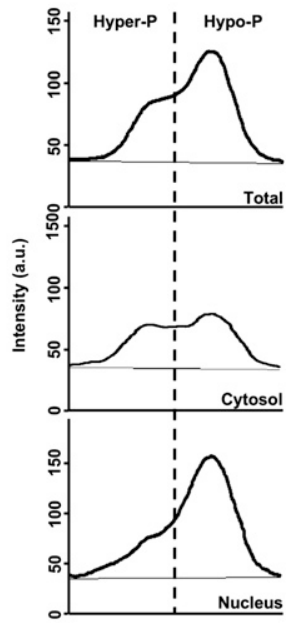

Figure 2. Phosphorylation state of FRQ affects shuttling kinetics. $(A)$ Western blot showing FRQ-FRB species with different phosphorylation states. (LI) Mycelia were grown $10 \mathrm{~h}$ in DD and then exposed to a 2-h light pulse to rapidly induce de novo synthesis of FRQ-FRB. (DD6) Light-grown mycelia were transferred to darkness for $6 \mathrm{~h}$ to shut down synthesis and allow progressive phosphorylation of previously synthesized FRQ-FRB. (CHX) Light-grown mycelia were treated $2 \mathrm{~h}$ with $10 \mu \mathrm{g} / \mathrm{mL} \mathrm{CHX}$ to induce hyperphosphorylation of FRQ-FRB. Heterogeneously phosphorylated FRQ of light-grown mycelia (LL) is shown for comparison. (B) Shuttling dynamics of hypophosphorylated and hyperphosphorylated FRQ-FRB. LI, DD6, and CHX cultures of frq-frb/h1-fkbp were incubated with $200 \mathrm{nM}$ rapamycin. Mycelia were harvested at the indicated times and fractionated. (Bottom panels) Quantification of the concentration of nuclear FRQ-FRB. Total and nuclear FRQ-FRB signals were normalized to the amount of protein $(200 \mu \mathrm{g}$ total, 100 $\mu \mathrm{g}$ of nuclei) and the ratio of nuclear versus total FRQ was plotted. Error bars represent SEM $(n=4)$. $(C)$ The CK1 inhibitor PF670462 interferes with hyperphosphorylation, cytoplasmic relocalization, and degradation of FRQ-FRB. Mycelia were treated for 0 and $2 \mathrm{~h}$ with CHX in the presence or absence of $15 \mu \mathrm{M}$ PF670462. Then, rapamycin was added for 30 min and samples were fractionated. (D) Subcellular distribution of "young" and "old" FRQ-FRB. Mycelia (DD6) were exposed to a 2-h light pulse. Rapamycin was added (10 $\mathrm{min}$ ) and the mycelia were fractionated. Hypophosphorylated "young" and hyperphosphorylated "old" FRQ-FRB are indicated by open and filled arrowheads, respectively. $(E, F)$ Example of densitometric analysis $(E)$ and quantification of the subcellular distribution $(F)$ of hypophosphorylated and hyperphosphorylated FRQ-FRB. Cytosolic and nuclear FRQ-FRB signals were normalized to the amount of protein $\left(200 \mu \mathrm{g}\right.$ of cytosol, $100 \mu \mathrm{g}$ of nuclei). Error bars represent SEM $(n=12) .\left(^{\star \star \star}\right) P<0.01$ (Student's $t$-test).

subsequently added CHX. After 2 h, FRQ-FRB was hyperphosphorylated, and when mycelia were transferred to FK506-containing medium, FRQ-FRB redistributed to the cytosol with similar kinetics as in the absence of CHX (Supplemental Fig. 3, right). This indicates that hyperphosphorylation does not inhibit nuclear export of FRQ-FRB. The kinetics of cytoplasmic relocalization of FRQ-FRB includes uptake of FK506 and dissociation of the stable rapamycin-induced ternary complex. Since these steps could be rate-limiting, we could not determine whether the kinetics of export is affected by the phosphorylation state of FRQ-FRB.

\section{Phosphorylation and subcellular localization of FRQ}

To test how nuclear localization of FRQ is affected by progressive phosphorylation, we monitored the temporal and spatial dynamics of a pulse of newly synthesized FRQ in comparison with previously synthesized FRQ. To distinguish between "young" and "old" FRQ, we constructed the strain $\mathrm{Fr}^{+}$, qa-frq-Flag, which expressed FRQ from its endogenous, light-inducible promoter and, in addition, a Flag-tagged version of FRQ (FRQ-Flag) under control of the inducible $q a-2$ promoter. The strain was initially grown in constant light in fructose medium to 
allow accumulation of steady-state levels of endogenous FRQ. Expression of FRQ-Flag was then induced for $4 \mathrm{~h}$ with quinic acid (QA) and subsequently repressed with glucose. Total and Flag-tagged FRQs were analyzed 0, 4, and $6 \mathrm{~h}$ after the glucose shift. Total FRQ was heterogeneously phosphorylated and localized predominantly in the cytosol at all time points (Fig. 3A, left). This observation shows that the system was in steady state. The induced FRQ-Flag, which is also recognized by the FRQ antibody, did not contribute detectably to the overall FRQ level, suggesting that FRQ synthesis from the endogenous gene was more efficient than from the qa-2driven gene. Immediately after the QA pulse, FRQ-Flag was rather hypophosphorylated and overrepresented in the nucleus (Fig. 3A, right). After $6 \mathrm{~h}$, FRQ-Flag had acquired a high phosphorylation state. The concentration ratio of nuclear to cytoplasmic FRQ-Flag (N/C concentration ratio) decreased, while the $\mathrm{N} / \mathrm{C}$ concentration ratio of total FRQ remained constant (Fig. 3B). A corresponding redistribution was observed when FRQ expression was induced under control of the $q a-2$ promoter in a frq-null background (Supplemental Fig. 4). The data demonstrate that newly synthesized FRQ undergoes a time-dependent maturation process independent of previously synthesized "old" FRQ. In the course of this maturation process FRQ is progressively phosphorylated, loses nuclear import competence, and is committed to degradation.

\section{Subcellular dynamics of mutant FRQ}

The mutant $f r q^{7}$ strain displays a long free-running period of $28 \mathrm{~h}$. It encodes, due to a point mutation, a FRQ ${ }^{7}$ protein with a glycine-to-aspartic acid exchange at position 459 (G459D). Upon LD transfer of mycelia, FRQ ${ }^{7}$ is turned over with reduced kinetics (Ruoff et al. 2005). We compared the subcellular distribution of FRQ and FRQ ${ }^{7}$. In LL (steady state), FRQ and FRQ ${ }^{7}$ were both heterogeneously phosphorylated to a similar extent and displayed a similar distribution between cytosol and nuclei (Fig. 4A). The mycelia were then transferred to the dark to shut down synthesis of FRQ and allow phosphorylation of the previously synthesized clock proteins. Six hours after the transfer (DD6) FRQ was essentially cleared from nuclei in wild type (Fig. 4A, left). This supports that hyperphosphorylation of FRQ interferes with nuclear localization. However, $\mathrm{FRQ}^{7}$ was present in nuclei to a similar extent at DD6 and in LL (Fig. 4A, right). We then asked whether the prolonged nuclear localization of FRQ $^{7}$ correlates with reduced kinetics of phosphorylation. For this purpose dark-grown wild type and $\mathrm{fr}^{7}$ were exposed at DD12 and DD16, respectively (minimum of FRQ and $\mathrm{FRQ}^{7}$ levels), to a 2 -h light pulse to rapidly induce synthesis of a wave of FRQ and FRQ ${ }^{7}$. Subsequently, samples were analyzed over a time course of $6 \mathrm{~h}$. FRQ was progressively hyperphosphorylated, while the phosphorylation kinetics of $\mathrm{FRQ}^{7}$ was reduced (Fig. 4B).

As expected, the nuclear levels of FRQ decreased in the course of the 6-h incubation in DD (Fig. 4C, left). In contrast, $\mathrm{FRQ}^{7}$ remained in a rather hypophosphorylated
A

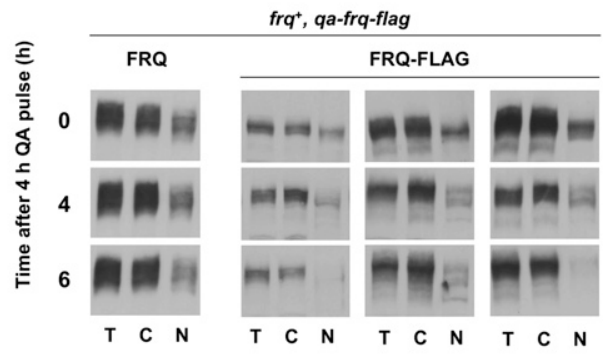

B

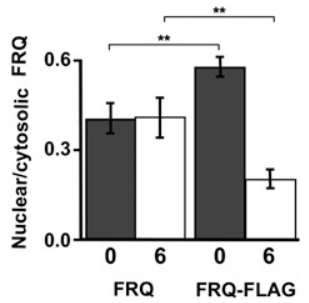

Figure 3. Phosphorylation and subcellular distribution of FRQ. (A) Phosphorylation-dependent maturation and subcellular redistribution of newly synthesized "young" FRQ are independent of previously synthesized "old" FRQ. Mycelia of frq", qa-frqFlag were grown in LL to allow accumulation of high steadystate levels of endogenous FRQ. Synthesis of FRQ-Flag was then induced for $4 \mathrm{~h}$ with $1 \mathrm{mM}$ QA and subsequently terminated by transfer to medium without QA. Mycelia were harvested after the indicated time periods, fractionated, and analyzed. Western blots show overall FRQ levels (left) and QA-induced FRQ-Flag (right panels; three independent experiments shown). A total of $200 \mu \mathrm{g}$ of cytosol and nuclei were analyzed. $(B)$ Quantification of cytoplasmic and nuclear FRQ and FRQ-Flag. Error bars represent SEM $(n=5) .\left(^{\star \star}\right) P<0.05$ (Student's $t$-test).

state and nuclear $\mathrm{FRQ}^{7}$ did not relocalize to the cytosol within the 6-h time course of the experiment (Fig. 4C, right). Thus, the reduced phosphorylation kinetics of $\mathrm{FRQ}^{7}$ correlates with prolonged nuclear localization, supporting the notion that nuclear import of FRQ is regulated by phosphorylation. Since nuclear accumulation of $\mathrm{FRQ}^{7}$ should correlate with prolonged negative feedback, the long free-running period of $\mathrm{frq}^{7}$ might be a direct consequence of the altered nucleocytoplasmic shuttling kinetics of $\mathrm{FRQ}^{7}$.

\section{Modeling nucleocytoplasmic shuttling and maturation of FRQ}

Our data suggest that the subcellular distribution of FRQ reflects the steady state of nucleocytoplasmic shuttling, and that phosphorylation-dependent maturation of FRQ is associated with cytoplasmic accumulation and degradation. We formulated a mathematical model (Fig. 5A; Supplemental Material) in which we assumed 50 distinct phosphorylation states of FRQ (F1-F50). Rates for shuttling were estimated from the experimental data presented here, and degradation rates were estimated from previous data (Gorl et al. 2001; Ruoff et al. 2005). 
A

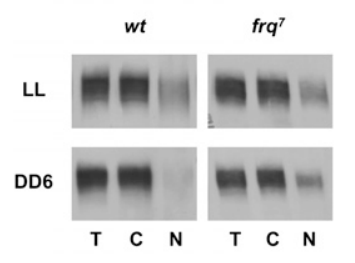

B

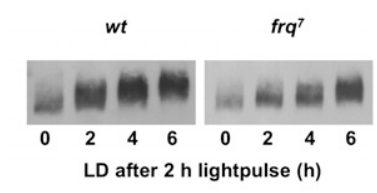

C

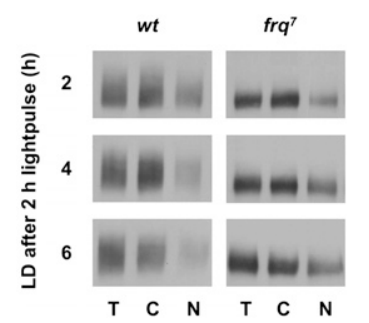

Figure 4. Subcellular dynamics of $\mathrm{FRQ}^{7}$. (A) Retarded nuclear clearance of $\mathrm{FRQ}^{7}$ after LD transfer. Light-grown wild type and $\mathrm{fr}^{7}$ were either kept in LL or transferred to the dark for $6 \mathrm{~h}$ (DD6) and mycelia were fractionated. $(B, C)$ Reduced phosphorylation kinetics and prolonged nuclear accumulation of $\mathrm{FRQ}^{7}$. Mycelia were initially grown in the dark and then exposed to a 2-h light pulse, and subsequently incubated in the dark for the indicated time periods. Samples were either directly analyzed $(B)$ or fractionated and analyzed $(C)$.

Specifically, we assumed that FRQ can adopt different conformations that depend on its phosphorylation state (Salazar and Hofer 2003, 2009). These conformations control nuclear import (e.g., via accessibility of a nuclear import signal) and degradation. Consequently, the rate of nuclear import of FRQ decreases, and its rate of degradation increases, as a sigmoid function of the degree of phosphorylation (Fig. 5B). The kinetics of export of FRQ was chosen to be independent of phosphorylation.

Rates of phosphorylation and dephosphorylation are difficult to estimate. When de novo synthesis of FRQ is inhibited, hypophosphorylated FRQ acquires an intermediate phosphorylation state in $\sim 2 \mathrm{~h}$ (Gorl et al. 2001; Ruoff et al. 2005). Considering the large number of phosphorylation sites in FRQ, phosphorylation and dephosphorylation of an individual site must occur in the range of seconds to minutes. We arbitrarily assumed that FRQ is rapidly phosphorylated with somewhat different rates in cytosol and nuclei. Since the overall phosphorylation state of FRQ increases progressively in the course of hours, phosphorylation must be slightly faster than dephosphorylation. Rates were optimized to fit the observed characteristics of FRQ and $\mathrm{FRQ}^{7}$ (Supplemental Material).

Using these parameters, FRQ and $\mathrm{FRQ}^{7}$ were constitutively synthesized for $22 \mathrm{~h}$. To mimic an LD transfer of mycelia, synthesis was then stopped for another $22 \mathrm{~h}$ (Fig.
5C). Phosphorylation levels, concentrations of nuclear and cytoplasmic protein, and the ratio of nuclear to cytosolic FRQ (Fig. 5C, solid and dotted black lines) and $\mathrm{FRQ}^{7}$ (Fig. 5C, solid and dotted gray lines) were computed. Following the induction of synthesis at $t=0$, FRQ was progressively phosphorylated and approached a steady state after $\sim 6 \mathrm{~h}$. In steady state, FRQ was heterogeneously phosphorylated and reached an average phosphorylation level of $\sim 0.4$ (i.e., on average only 20 of the 50 sites were phosphorylated). The hyperphosphorylated state was not strongly populated due to rapid degradation of these species (Supplemental Fig. 5A). Early after FRQ induction, when predominantly hypophosphorylated FRQ was present, the protein was enriched in the nuclei. The maximal N/C concentration ratio of FRQ was $\sim 0.7$. With increasing phosphorylation state, FRQ relocalized gradually to the cytosol. In steady state, FRQ was predominantly cytosolic and the N/C concentration ratio was 0.4 , which reflects approximately the ratio observed in vivo under steady-state conditions in LL (see Fig. 3B). When production of FRQ was stopped $(t=22)$, FRQ was degraded. The average phosphorylation level of FRQ increased within $\sim 6 \mathrm{~h}$ from 0.4 to 0.5 , and the nuclear ratio of FRQ decreased more than twofold during the degradation phase (Fig. 5C). A corresponding behavior of FRQ was observed in vivo upon LD transfer of mycelia (see Fig. 4C, left).

FRQ $^{7}$ was phosphorylated with reduced kinetics, but approached a similar phosphorylation level in steady state. Overall expression levels of $\mathrm{FRQ}^{7}$ were higher than those of FRQ, which is also observed in vivo (Ruoff et al. 2005). Newly synthesized FRQ ${ }^{7}$ accumulated like FRQ in the nuclei and approached a similar subcellular distribution in steady state. When synthesis was stopped, $\mathrm{FRQ}^{7}$ was turned over with slow kinetics in agreement with experimental observations (see Fig. 4C, right panels). It should be noted that we changed only the phosphorylation rate, but not the degradation rate per se. As observed in vivo (see Fig. 4A), the N/C concentration ratio of $\mathrm{FRQ}^{7}$ remained rather high in silico (Fig. 5C). Thus, the model predicted the characteristics of wild-type and mutant FRQ.

To mimic intrinsic circadian rhythms, we then simulated oscillatory synthesis of FRQ with a period of $22 \mathrm{~h}$ (Fig. 5D). The ratio of nuclear to cytosolic FRQ did not stay constant, but oscillated with an advanced phase with respect to the circadian rhythm of FRQ accumulation. The maximal N/C concentration ratio coincided with the minimal phosphorylation level of FRQ. Hence, a model based on rapid rates of shuttling and phosphorylation reproduces the observed circadian dynamics of subcellular distribution of FRQ on a circadian time scale (Luo et al. 1998; Schafmeier et al. 2006).

Finally, we measured experimentally, and simulated in silico, the subcellular distribution of FRQ in steady state at different temperatures. Neurospora was grown in LL at $20^{\circ} \mathrm{C}, 25^{\circ} \mathrm{C}$, and $30^{\circ} \mathrm{C}$, and mycelia were fractionated. As reported, expression levels of FRQ depend strongly on temperature due to complex regulation of frq RNA splicing and translation (Colot et al. 2005; Diernfellner et al. 2005). However, the subcellular distribution of FRQ 
A

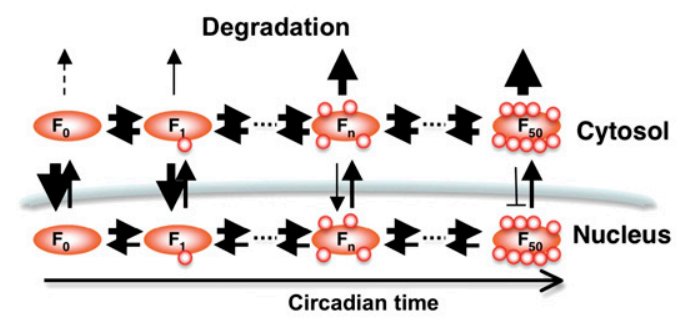

B

Figure 5. Mathematical model of phosphorylationdependent maturation of FRQ. (A) Schematic presentation of nucleocytoplasmic shuttling, phosphorylation, and degradation of FRQ. Newly synthesized, unphosphorylated FRQ (FO) is progressively phosphorylated in 50 steps (F50). Phosphorylation is fast and is antagonized by rapid dephosphorylation. FRQ shuttles between nucleus and cytosol and eventually is degraded. With increasing phosphorylation state, the degradation rates of FRQ increase and nuclear import rates decrease. Export of FRQ is independent of phosphorylation. For details, see the Supplemental Material. (B) Assumed dependence of degradation rate (left) and nuclear import rate (right) of FRQ on its phosphorylation state. $(C, D$, top panels) FRQ was either synthesized at a constant rate for $22 \mathrm{~h}$ and synthesis was then stopped, or rhythmically synthesized with a period of $22 \mathrm{~h}$. Accumulation, average phosphorylation level, and subcellular FRQ concentrations (middle panels) and N/C concentration ratios (bottom panels) were calculated.
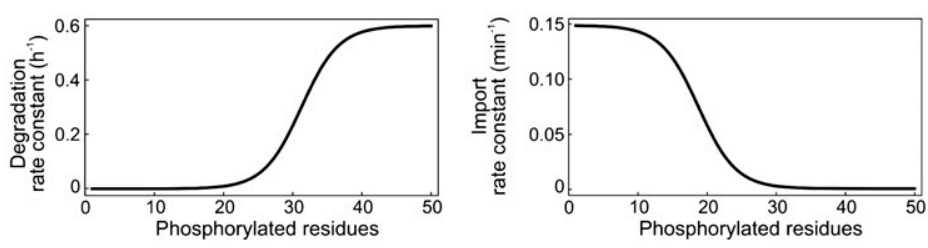

C

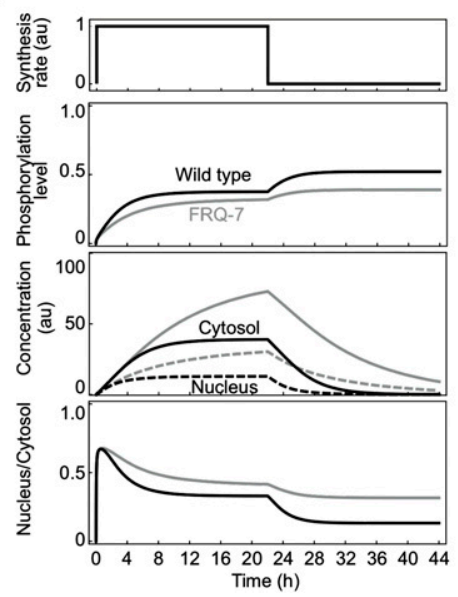

D

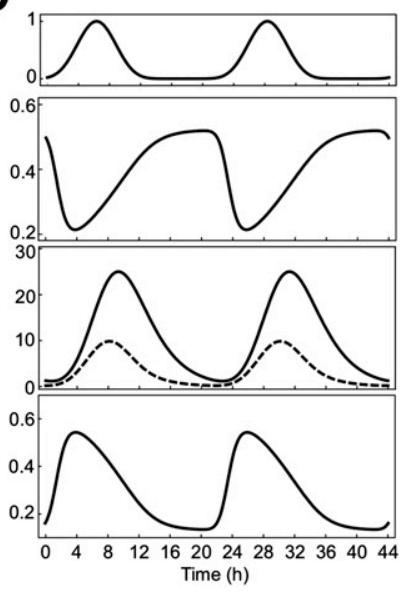

displayed only weak temperature dependence (Supplemental Fig. 5B). To simulate temperature shifts in silico, we increased or decreased the rates of FRQ shuttling, phosphorylation, and dephosphorylation. The degradation rate of FRQ, which depends only weakly on temperature (Ruoff et al. 2005), was kept constant. The predicted nucleocytoplasmic distribution of FRQ was essentially independent of the shuttling and phosphorylation rates and similar to the in vivo distribution of FRQ at different temperatures (Supplemental Fig. 5C). These simulations suggest that fast kinetics of phosphorylation and shuttling compensate the nucleocytoplasmic distribution of FRQ against temperature changes.

\section{Discussion}

How the interaction of clock components faithfully produces robust biochemical oscillations on a circadian time scale is a crucial question in the field of circadian clocks. Here we showed that the subcellular redistribution of FRQ on a circadian time scale is governed by rapid, noncircadian cycles of nuclear import and export. The rate of nuclear import of newly synthesized FRQ is progressively reduced in a phosphorylation-dependent manner, leading to an increase in steady-state levels of cytoplasmic FRQ. We also present a mathematical model that describes the cytoplasmic accumulation of wild-type and mutant FRQ on a circadian time scale on the basis of frequencymodulated rapid nucleocytoplasmic shuttling cycles.

We found that the observed subcellular distribution of FRQ reflects the steady state of nucleocytoplasmic shuttling. The shuttling kinetics of FRQ correlates with its phosphorylation state. Hypophosphorylated FRQ is imported readily into nuclei, whereas import of hyperphosphorylated FRQ is compromised.

FRQ is phosphorylated at $>70$ sites (Baker et al. 2009; Tang et al. 2009). It seems unlikely that all of these sites are phosphorylated in a strictly ordered fashion and that each phosphorylation event triggers a distinct function. Rather, hyperphosphorylation may alter protein surface charge and structure and thereby progressively affect a few specific functions of FRQ, including subcellular shuttling. 
We described the dynamics of subcellular localization of FRQ in a mathematical model. The model reproduces experimental observations, supporting that the observed subcellular distribution of FRQ reflects the steady state of nucleocytoplasmic shuttling. Each individual molecule of FRQ undergoes a phosphorylation-dependent maturation process independent of other FRQ molecules. Thus, FRQ can be viewed as a molecular hourglass. In the course of maturation, FRQ gradually loses import competence, resulting in an increase of cytosolic FRQ levels. We showed recently that cytosolic accumulation of FRQ is crucial for the positive feedback loop of the clock, since FRQ-dependent phosphorylation of WCC in the cytosol interferes with nuclear import of the transcription factor and thereby supports accumulation of inactive and stable WCC (Schafmeier et al. 2008).

Expression levels of FRQ depend strongly on temperature (Liu et al. 1997) and are regulated in a complex manner on the level of thermosensitive splicing and translation (Colot et al. 2005; Diernfellner et al. 2007). Although temperature should affect the kinetics of shuttling and phosphorylation, we found that the ratio of nuclear to cytosolic FRQ is essentially independent of temperature, indicating that the coordination of FRQ functions in both compartments might be important. Interestingly, the model suggests that the steady state of the subcellular ratio of FRQ is essentially independent of the rates of FRQ shuttling and phosphorylation as long as these processes are much faster than the degradation rate. Hence, fast shuttling and phosphorylation cycles might be important for the robustness of the system toward perturbations and might contribute to temperature compensation of the clock.

Changes in nucleocytoplasmic localization of clock components on a circadian time scale has been observed in mammals and flies and is crucial for clock function in these systems, and evidence is accumulating that these processes can be regulated by post-translational modifications such as phosphorylation (Tamanini et al. 2005). In flies, the circadian transcription factors CLK and CYC have been reported to shuttle between nucleus and cytosol (Hung et al. 2009). Furthermore, nuclear accumulation of PER and TIM is regulated by CK1 and by dissociation of cytosolic comlexes (Meyer et al. 2006). In mammals, BMAL1 (Tamaru et al. 2003; Kwon et al. 2006) and PER2 (Yagita et al. 2002) were shown to use classical nuclear localization signals (NLS) and nuclear export signals (NES) to shuttle between cytosol and nuclei. It would be interesting to see whether the apparent circadian dynamics of subcellular relocalization of these clock components are governed by phosphorylation and modulation of rapid shuttling cycles, similar to FRQ in Neurospora.

\section{Materials and methods}

Neurospora strains and culture conditions

Neurospora strains carried the ras- $1^{\text {bd }}$ mutation (Belden et al. 2007). For transformations, ras- $1^{\text {bd }}(w t)$; fr $q^{10}$; his-3 and ras- $1^{\text {bd }}$; $\mathrm{frq}^{+}$; his-3 were used. Standard growth medium contained $2 \%$

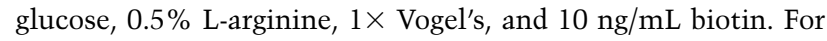
QA induction, glucose was replaced by fructose, and $1 \mathrm{mM}$ QA was added. In race tube medium, the glucose content was reduced to $0.1 \%$ and arginine was reduced to $0.17 \%$. Analysis of race tubes was performed using the ChronoII program (T. Roenneberg, Ludwig-Maximilians-Universität, Munich). When indicated, $10 \mu \mathrm{g} / \mathrm{mL}$ CHX, $200 \mathrm{nM}$ rapamycin, and $10 \mu \mathrm{M}$ FK506 were used.

\section{Plasmid construction and Neurospora transformation}

Construction of the vector containing frq-frb and h1-fkbp is described in the Supplemental Material. Neurospora conidia were transformed by electroporation as described (Schafmeier et al. 2006). The his-3 locus was used for homologous recombination. Insertion of the target gene was verified by PCR and sequencing.

\section{Protein analysis}

Extraction of Neurospora protein, two-deimensional electrophoresis, and dephosphorylation of extracts were performed as described (Diernfellner et al. 2005; Schafmeier et al. 2005). The procedure of subcellular fractionation is described in the Supplemental Material. Proteins $(200 \mu \mathrm{g}$ of total extract or cytosol, or $100 \mu \mathrm{g}$ of nuclei, determined by Bradford assay) were subjected to SDS-PAGE and Western blotting. Enhanced chemiluminescence signals were detected with X-ray films. Series of exposures were generated for quantification of bands.

\section{Acknowledgments}

We thank Sabine Schultz for excellent technical assistance and Tobias Schafmeier for critical reading of the manuscript. This work was supported by Deutsche Forschungsgemeinschaft grants BR 1375-1 and SFB 638. M.B and T.H. are members of CellNetworks.

\section{References}

Aronson BD, Johnson KA, Loros JJ, Dunlap JC. 1994. Negative feedback defining a circadian clock: Autoregulation of the clock gene frequency. Science 263: 1578-1584.

Badura L, Swanson T, Adamowicz W, Adams J, Cianfrogna J, Fisher K, Holland J, Kleiman R, Nelson F, Reynolds L, et al. 2007. An inhibitor of casein kinase I $\varepsilon$ induces phase delays in circadian rhythms under free-running and entrained conditions. I Pharmacol Exp Ther 322: 730-738.

Baker CL, Kettenbach AN, Loros JJ, Gerber SA, Dunlap JC. 2009. Quantitative proteomics reveals a dynamic interactome and phase-specific phosphorylation in the Neurospora circadian clock. Mol Cell 34: 354-363.

Banaszynski LA, Liu CW, Wandless TJ. 2005. Characterization of the FKBP.rapamycin.FRB ternary complex. I Am Chem Soc 127: 4715-4721.

Belden WJ, Larrondo LF, Froehlich AC, Shi M, Chen CH, Loros JI, Dunlap JC. 2007. The band mutation in Neurospora crassa is a dominant allele of ras-1 implicating RAS signaling in circadian output. Genes \& Dev 21: 1494-1505.

Brunner M, Kaldi K. 2008. Interlocked feedback loops of the circadian clock of Neurospora crassa. Mol Microbiol 68: 255262.

Bryant CD, Graham ME, Distler MG, Munoz MB, Li D, Vezina P, Sokoloff G, Palmer AA. 2009. A role for casein kinase $1 \varepsilon$ in the locomotor stimulant response to methamphetamine. Psychopharmacology 203: 703-711. 
Cha J, Chang SS, Huang G, Cheng P, Liu Y. 2008. Control of WHITE COLLAR localization by phosphorylation is a critical step in the circadian negative feedback process. EMBO J 27: 3246-3255.

Cheng P, Yang Y, Liu Y. 2001. Interlocked feedback loops contribute to the robustness of the Neurospora circadian clock. Proc Natl Acad Sci 98: 7408-7413.

Colot HV, Loros JJ, Dunlap JC. 2005. Temperature-modulated alternative splicing and promoter use in the Circadian clock gene frequency. Mol Biol Cell 16: 5563-5571.

Diernfellner AC, Schafmeier T, Merrow MW, Brunner M. 2005. Molecular mechanism of temperature sensing by the circadian clock of Neurospora crassa. Genes \& Dev 19: 1968 1973.

Diernfellner A, Colot HV, Dintsis O, Loros JJ, Dunlap JC, Brunner M. 2007. Long and short isoforms of Neurospora clock protein FRQ support temperature-compensated circadian rhythms. FEBS Lett 581: 5759-5764.

Dunlap JC, Loros JJ, Colot HV, Mehra A, Belden WJ, Shi M, Hong CI, Larrondo LF, Baker CL, Chen CH, et al. 2007. A circadian clock in Neurospora: How genes and proteins cooperate to produce a sustained, entrainable, and compensated biological oscillator with a period of about a day. Cold Spring Harb Symp Quant Biol 72: 57-68.

Gorl M, Merrow M, Huttner B, Johnson J, Roenneberg T, Brunner M. 2001. A PEST-like element in FREQUENCY determines the length of the circadian period in Neurospora crassa. EMBO J 20: 7074-7084.

Haruki H, Nishikawa J, Laemmli UK. 2008. The anchor-away technique: Rapid, conditional establishment of yeast mutant phenotypes. Mol Cell 31: 925-932.

Hong CI, Ruoff P, Loros JJ, Dunlap JC. 2008. Closing the circadian negative feedback loop: FRQ-dependent clearance of WC-1 from the nucleus. Genes \& Dev 22: 196-204.

Hung HC, Maurer C, Zorn D, Chang WL, Weber F. 2009. Sequential and compartment specific phosphorylation controls the life cycle of the circadian CLOCK protein. I Biol Chem 284: 23734-23742.

Ko CH, Takahashi JS. 2006. Molecular components of the mammalian circadian clock. Hum Mol Genet 15: R271R277. doi: 10.1093/hmg/ddl207.

Kwon I, Lee J, Chang SH, Jung NC, Lee BJ, Son GH, Kim K, Lee KH. 2006. BMAL1 shuttling controls transactivation and degradation of the CLOCK/BMAL1 heterodimer. Mol Cell Biol 26: 7318-7330.

Lee K, Loros JJ, Dunlap JC. 2000. Interconnected feedback loops in the Neurospora circadian system. Science 289: 107-110.

Liu Y, Bell-Pedersen D. 2006. Circadian rhythms in Neurospora crassa and other filamentous fungi. Eukaryot Cell 5: 1184 1193.

Liu Y, Garceau NY, Loros JJ, Dunlap JC. 1997. Thermally regulated translational control of FRQ mediates aspects of temperature responses in the Neurospora circadian clock. Cell 89: 477-486.

Luo C, Loros JJ, Dunlap JC. 1998. Nuclear localization is required for function of the essential clock protein FRQ. EMBO J 17: 1228-1235.

Merrow M, Roenneberg T. 2007. Circadian entrainment of Neurospora crassa. Cold Spring Harb Symp Quant Biol 72: 279-285.

Meyer P, Saez L, Young MW. 2006. PER-TIM interactions in living Drosophila cells: An interval timer for the circadian clock. Science 311: 226-229.

Roenneberg T, Merrow M. 2007. Entrainment of the human circadian clock. Cold Spring Harb Symp Quant Biol 72: 293299.
Rosbash M, Bradley S, Kadener S, Li Y, Luo W, Menet JS, Nagoshi E, Palm K, Schoer R, Shang Y, et al. 2007. Transcriptional feedback and definition of the circadian pacemaker in Drosophila and animals. Cold Spring Harb Symp Quant Biol 72: 75-83.

Ruoff P, Loros JJ, Dunlap JC. 2005. The relationship between FRQ-protein stability and temperature compensation in the Neurospora circadian clock. Proc Natl Acad Sci 102: 1768117686.

Salazar C, Hofer T. 2003. Allosteric regulation of the transcription factor NFAT1 by multiple phosphorylation sites: A mathematical analysis. J Mol Biol 327: 31-45.

Salazar C, Hofer T. 2009. Multisite protein phosphorylationfrom molecular mechanisms to kinetic models. FEBS J 276: 3177-3198.

Schafmeier T, Haase A, Kaldi K, Scholz J, Fuchs M, Brunner M. 2005. Transcriptional feedback of Neurospora circadian clock gene by phosphorylation-dependent inactivation of its transcription factor. Cell 122: 235-246.

Schafmeier T, Kaldi K, Diernfellner A, Mohr C, Brunner M. 2006. Phosphorylation-dependent maturation of Neurospora circadian clock protein from a nuclear repressor toward a cytoplasmic activator. Genes \& Dev 20: 297-306.

Schafmeier T, Diernfellner A, Schafer A, Dintsis O, Neiss A, Brunner M. 2008. Circadian activity and abundance rhythms of the Neurospora clock transcription factor WCC associated with rapid nucleo-cytoplasmic shuttling. Genes \& Dev 22: 3397-3402.

Tamanini F, Yagita K, Okamura H, van der Horst GT. 2005. Nucleocytoplasmic shuttling of clock proteins. Methods Enzymol 393: 418-435.

Tamaru T, Isojima Y, van der Horst GT, Takei K, Nagai K, Takamatsu K. 2003. Nucleocytoplasmic shuttling and phosphorylation of BMAL1 are regulated by circadian clock in cultured fibroblasts. Genes Cells 8: 973-983.

Tang CT, Li S, Long C, Cha J, Huang G, Li L, Chen S, Liu Y. 2009. Setting the pace of the Neurospora circadian clock by multiple independent FRQ phosphorylation events. Proc Natl Acad Sci 106: 10722-10727.

Vitalini MW, Morgan LW, March IJ, Bell-Pedersen D. 2004. A genetic selection for circadian output pathway mutations in Neurospora crassa. Genetics 167: 119-129.

Yagita K, Tamanini F, Yasuda M, Hoeijmakers JH, van der Horst GT, Okamura H. 2002. Nucleocytoplasmic shuttling and mCRY-dependent inhibition of ubiquitylation of the mPER2 clock protein. EMBO J 21: 1301-1314. 


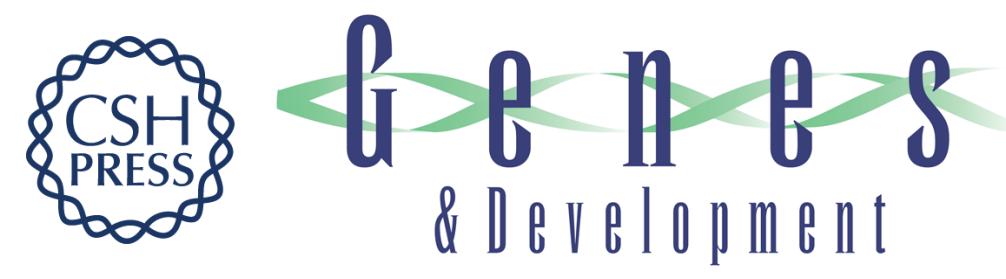

\section{Phosphorylation modulates rapid nucleocytoplasmic shuttling and cytoplasmic accumulation of Neurospora clock protein FRQ on a circadian time scale}

Axel C.R. Diernfellner, Christina Querfurth, Carlos Salazar, et al.

Genes Dev. 2009, 23:

Access the most recent version at doi:10.1101/gad.538209

Supplemental Material

References License

Email Alerting Service
http://genesdev.cshlp.org/content/suppl/2009/09/16/23.18.2192.DC1

This article cites 38 articles, 23 of which can be accessed free at: http://genesdev.cshlp.org/content/23/18/2192.full.html\#ref-list-1

Receive free email alerts when new articles cite this article - sign up in the box at the top right corner of the article or click here.

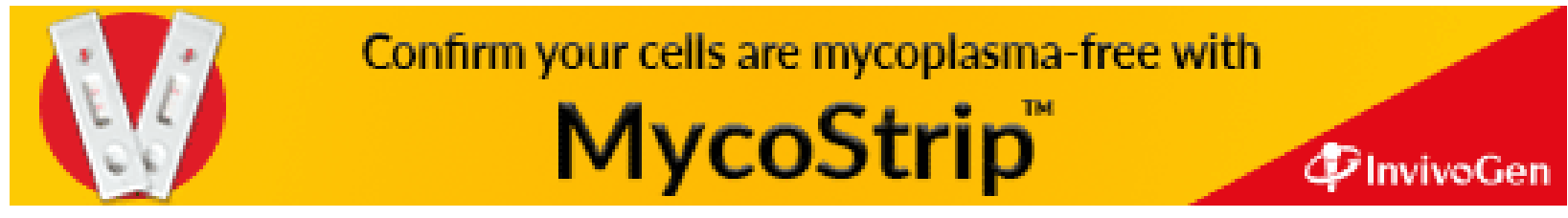

